\title{
Biases and Their Effects in Inductive Logic Programming
}

\author{
Birgit Tausend \\ Fakultät Informatik, Universität Stuttgart, Breitwiesenstr. 20-22, D-70565 Stuttgart
}

\begin{abstract}
The shift from attribute-value based hypothesis languages to Horn clause logic as in Inductive Logic Programming (ILP) results in a very complex hypothesis space. In this paper, we study how the basic constituents of biases reduce the size of the hypothesis space in ILP.
\end{abstract}

\section{Introduction}

Inductive logic programming (ILP) [Mug93] aims to overcome the limitations of attribute-value based learning algorithms by using Horn logic as hypothesis language. However, a powerful representation language like Horn logic accounts for a very complex hypothesis space. This complexity may prevent systems from finding an appropriate hypothesis in a reasonable time or at all. As a consequence, there is a growing interest in appropriate biases to control the search space, in particular in language biases.

In this paper, we study the influence of basic bias constiutents on the complexity of the hypothesis space. As the measure of theory costs, introduced in [PK92], only applies to a restricted subset of hypotheses languages, we first extend it to unrestricted Horn logic. Using the new measure, we show how the upper bound of theory costs varies with respect to the bias constituents.

\section{Complexity of the Hypothesis Language in ILP}

The inductive logic programming problem is to find a logic program $H$ given the positive examples $E^{+}$, the negative examples $E^{-}$and the background knowledge $B$ [Mug93]. $H$ is a set of definite clauses $C: l_{0} \leftarrow l_{1}, \ldots, l_{n}$ in a hypothesis language $L_{H}$, and has to be necessary, i.e. $B \forall E^{+}$, sufficient, i.e. $B \wedge H \vdash E^{+}$, and consistent, i.e. $B \wedge H \wedge E^{-} \forall \square$.

The total number of literals to be considered by FOIL [Qui90] for specializing a hypothesis clause with $k$ variables can be measured by the so called theory costs $C_{T H_{F O I L}}$. These costs depend on the number of variabilizations $v(i, k)$, i.e. the choice of variables for the predicate of arity $i$ [PK92], and they are given by:

$$
C_{T H_{F O I L}}=2 * \sum_{i=1}^{M a x A} \operatorname{Pred}(i) * v(i, k)
$$

where $k \geq 1, \operatorname{pred}(i)$ is the number of predicates of arity $i, \operatorname{Max} A$ is the maximum arity of any predicate, and the factor 2 indicates that both positive and negative literals may occur. 
An upper bound of $C_{T H_{F O I L}}$ with respect to the maximum number of old variables Old and the total number of predicates AllPred is given by [PK92]:

$$
C_{T H_{F O I L}} \leq 2 * \text { AllPred } *(\text { Old }+\operatorname{Max} A-1)^{M a x A} \text {. }
$$

As $C_{T H_{F O I L}}$ applies only to hypothesis languages with function free linked clauses as in FOIL [Qui90], we define a similar measure $C_{T H}$ for languages of unrestricted Horn clauses. We replace the variabilizations $v(i, k)$ by term distributions $t(i, k)$, i.e. the mapping of $k$ old and $i$ new terms to the arguments positions of a predicate. In contrast to a variabilization, a term distribution $t(i, k)$ takes into account structured terms and constants as well. An upper bound of theory costs of unrestricted Horn clauses as hypothesis language is given by

$$
C_{T H} \leq 2 * \text { AllPred } *(\text { OldTerms }+ \text { NewTerms })^{M a x A},
$$

where OldTerms is the number of terms in the hypothesis to be specialized and NewTerms is the number of new terms in the new literal.

\section{Effects of Biases Constituents}

Restricting a hypothesis language $L_{H}$ means for example reducing $C_{T H}$ by excluding negated literals, or decreasing the parameters of $C_{T H}$, the total number of predicates AllPred, the number of old and new terms, OldTerms and NewTerms, and the maximum arity $\operatorname{Max} A$. Another way to reduce $C_{T H}$ is to restrict the mapping of terms to argument positions, i.e. the term distributions.

\subsection{Reducing MaxA and AllPred}

In ILP, the total number of predicates AllPred and the maximum arity MaxA are restricted to the predicates occurring in the background knowledge $B$ and the examples $E$. Only few systems include capabilites to extend these numbers by invented predicates.

Similarly, the parameters $M a x A$ and AllPred are only restricted in few ILP systems. For example, excluding commutative variants of a literal as in [PK92] reduces AllPred. Another bias constituent reducing AllPred is to allow only predicates of a certain type, e.g. numeric or list predicates, in the new literal, as in e.g. MOBAL [MWKE93] or the recent version of FOIL [QCJ93].

\subsection{Reducing the Number of Terms}

Both OldTerms and NewTerms are subject to several bias constituents in ILP, as the number of terms Terms $=$ OldTerms $+N e w T e r m s$ strongly influences the size of $L_{H}$. For example, in [PK92] it is shown that $C_{T H_{F O I L}}$ increases exponentially by the number of variables.

The number of terms may be infinite in Horn logic as terms are constructed by applying functors in the signature to other terms. However, the construction of terms for a hypothesis clause of ILP is often restricted either to use only functors and constants occurring in the examples, or to avoid term construction by excluding structured terms not occurring as terms and subterms in the examples. This leads to a finite number of terms Terms. 
A widely used restriction on terms is to use function-free Horn logic, e.g. in FOIL [Qui90], CLINT [DR91] or MOBAL [MWKE93]. This restriction strongly reduces $C_{T H}$ as it excludes literals including n-place functors where $n \geq 1$. The number of terms is Terms $=(O l d V C+N e w V C)$ in a function-free language, and OldVC is the number of old variables and constants and NewVC the number of new variables and constants. Similar results can be achieved by using constant-free Horn logic.

Additional restrictions of function free languages mainly reduce the number of new variables in a literal not occurring in the preceeding literals in the clause.

The number of new variables can be limited to a fixed number $i$ as in some languages series of CLINT [DR91], i.e. Terms $=$ OldVC $+i$. Other language series vary this number with respect to the depth of the new literal. Then, Terms $=$ OldVC $+i(d)$, and $i(d)$ is the number of new variables at depth $i$. The depth is defined by the maximum length of a connection path established by shared variables.

Definition 1. Let $C: l_{0} \leftarrow l_{1}, \ldots, l_{n}$ be a clause. The depth $\operatorname{depth}(X)$ of a variable $X$ is defined by $\operatorname{depth}(X)=\min \{\operatorname{length}(\operatorname{co}(X)) \mid c p(X)$ is a connection path of $X$ \}, and $c p(X)$ is a connection path as in definition 3. The depth of a clause $C$ (a literal $l_{i}$ ) is the maximum depth of its variables.

The restriction to constrained clauses sets the number of new variables to 0 , i.e. $T e r m s=O l d V C$, and the body literals only include variables of the head.

Another basic constituent reducing Terms is to exclude unlinked clauses, as in ITOU [Rou92], CLINT [DR91], GOLEM [MF90], SIERES [WO91].

Definition 2. A clause $C: l_{0} \leftarrow l_{1}, \ldots, l_{n}$ is linked if all of its literals are linked. A literal $l_{i}$ is linked if it $\left\{\begin{array}{l}\text { is the head literal of } C \text {, i.e. } i=0 \\ \text { contains a variable } X, X \in \operatorname{vars}\left(l_{j}\right) \text { and } l_{j} \text { is linked. }\end{array}\right.$

As excluding such literals does not change the success set of a clause ${ }^{1}$, they can be omitted. The linkedness restriction is very weak as it reduces the number of terms only by one, i.e. Terms $=($ OldVC $+N e w V C-1)$.

\subsection{Restricting the Mappings of Terms to Positions}

The maximum number $M a x T D$ of term distributions $t(i, k)$ given by $M a x T D=$ Terms ${ }^{M a x A}$ can be reduced by limiting the mapping of terms to argument positions without restricting the maximum number of terms Terms.

A basic bias constituent applied in ILP for this purpose are argument types, as in INDICO [STW93]. As a term may not occur at an argument position with conflicting type, $\operatorname{MaxTD}=\left(\operatorname{Terms}\left(\operatorname{Type}_{1}\right) * \ldots * \operatorname{Terms}\left(\operatorname{Type}_{\mathbf{M} a x A}\right)\right)$ where Terms $\left(\right.$ Type $\left._{i}\right)$ is the total number of old and new terms of argument type Type . $_{i}$. A bias constituent of a similar effect is given by mode declarations defining the arguments of a literal in terms of input and output arguments.

Another way to restrict $t(i, k)$ is to require unique variables, as in FOCL [PK92] or some languages of CLINT [DR91]. The number of terms Terms(i) at an argument position $i$ is reduced by the number of all terms including variables that already occur at other argument positions.

\footnotetext{
${ }^{1}$ except in the failure case
} 
The range restriction, e.g. in CLINT [DR91] or GOLEM [MF90], also reduces $M a x T D$ as all head variables must occur in the body literals.

A boundary case of restricting the mapping is given by the rule models [MWKE93] as the term distribution is fixed. Consequently, the number of term distributions is limited to the set of instantiable literals in all partially instantiated rule models.

Graph-based approaches, as described in e.g. [WO91], weaken this restriction by specifying particular connection paths by their edges.

Definition 3. A connection path of a variable $X$ in a clause $C: l_{0}-l_{1}, \ldots, l_{n}$ is $c p(X)= \begin{cases}\emptyset & \text { if } i=0 \\ c p(Y) \circ l_{i} & \text { if } \exists Y \in \operatorname{vars}\left(l_{i}\right), Y \neq X, \text { and } c p(Y) \text { does not include } l_{i} .\end{cases}$

This restriction limits the mapping of terms to argument positions to mappings that do not conflict with specified the connection paths.

\section{Conclusions}

The basic constituents of biases in ILP mainly reduce the number of terms and term distributions. Using the measure of theory costs, upper bounds for these constituents can be defined. The restriction to function free Horn clauses is particularly useful as it limits the terms to a finite set. Controlling the complexity of languages that are not function free by extending constituents applicable to function free languages or by defining new ones should be subject to further research.

Acknowledgements This work has been partially supported by ESPRIT BRA 6020 ILP. I would like to thank Irene Stahl for comments on drafts of this paper.

\section{References}

[DR91] L. De Raedt. Interactive Concept-Learning. PhD thesis, Katholieke Universiteit Leuven, 1991.

[MF90] S. Muggleton and C. Feng. Efficient induction of logic programs. In First Conference on Algorithmic Learning Theory, Tokyo, 1990. Ohmsha.

[Mug93] S. Muggleton. Inductive logic programming: Derivations, successes and shortcoming. In Machine Learning: ECML-93 . Springer, 1993.

[MWKE93] K. Morik, S. Wrobel, J. Kietz, and W. Emde. Knowledge Aquisition and Machine Learning: Theory, Methods, and Applications. Academic P., 1994.

[PK92] M. Pazzani and D. Kibler. The utility of knowledge in inductive learning. Machine Learning, 9:57-94, 1992.

[QCJ93] J.R. Quinlan and R.M. Cameron-Jones. Foil: A midterm report. In Machine Learning: ECML-93 Learning . Springer, 1993.

[Qui90] J. R. Quinlan. Learning logical definitions from relations. Machine Learning, 5:239-266, 1990.

[Rou92] C. Rouveirol. Extensions of inversion of resolution applied to theory completion. In S. Muggleton (Ed.), Inductive logic programming. Academic Press., 1992.

[STW93] I. Stahl, B. Tausend, and R. Wirth. Two methods for improving inductive logic programming systems.In Machine Learning: ECML-93 Springer, 1993.

[WO91] R. Wirth and P. O'Rorke. Constraints on predicate invention. In Eighth International Conference on Machine Learning. Morgan Kaufmann, 1991. 